\title{
A LIPSCHITZIAN CHARACTERIZATION OF CONVEX POLYHEDRA
}

\author{
DAVID W. WALKUP AND ROGER J.-B. WETS
}

1. Introduction. Consider an affine transformation $\tau$ between two Euclidean spaces, say $E^{n}$ and $E^{m}$, taking a subset $K$ of $E^{n}$ onto $\tau(K)$ in $E^{m}$. For each point $y$ in $E^{m}$ the set $\kappa(y)=\tau^{-1}(y) \cap K$ is the intersection of $K$ and a translate of the null space $N$ of $\tau$ in $E^{n}$. The Hausdorff distance $d\left(\kappa(y), \kappa\left(y^{\prime}\right)\right)$ is a metric on the collection of all nonempty sections $\kappa(y)$ for given $K$ and $\tau$. It is convenient, and consistent, to define the distance between the empty set and a nonempty intersection to be $+\infty$. Then $\kappa$ is a mapping from the metric space $E^{m}$ onto the metric space of sections. We shall say that $\kappa$ is Lipschitzian (with constant $B$ ) if there exists a constant $B$, depending only on $K$ and $\tau$, such that

$$
d\left(\kappa(y), \kappa\left(y^{\prime}\right)\right) \leqq B\left\|y-y^{\prime}\right\|
$$

whenever $\kappa(y)$ and $\kappa\left(y^{\prime}\right)$ are nonempty.

The following definition and theorem, taken together, are the main results of this paper.

Definition 1. A subset $K$ of $E^{n}$ is said to have property $\Omega_{j}$, if for every affine transformation $\tau$ defined on $E^{n}$ with null space of dimension $j$, the inverse map $\kappa$ associated with $\tau$ and $K$ is Lipschitzian.

Theorem 1. Suppose $K$ is a closed convex subset of $E^{n}$ and $1 \leqq j$ $\leqq n-1$. Then $K$ is a polyhderon if and only if $K$ has property $\mathfrak{R}_{j}$.

Since the identity map of any finite-dimensional linear space $R^{k}$ with one norm onto $R^{k}$ with any other norm is Lipschitzian, Theorem 1 remains valid if $\tau$ is an affine transformation from $R^{n}$ with any norm onto $R^{n-i}$ with any other norm, rather than from $E^{n}$ onto $E^{n-i}$.

This theorem supplements the characterization of convex polyhedra in terms of the polyhedral properties of $j$-sections and $j$-projections obtained by Klee in [1]. The first three sections of [1] may also serve as an introduction to the basic properties of polyhedra, which will be assumed here. We remark that the idea of analyzing the properties of sections of convex sets in terms of a Lipschitzian property of the Hausdorff distance has also appeared in a paper by Klee [2] concerned with the existence of certain universal Banach spaces.

Received by the editors August 2, 1968. 
2. Polyhedra have property $\mathfrak{l}_{j}$. The Hausdorff distance between two nonempty subsets $S$ and $S^{\prime}$ of $E^{n}$ may be written as

$$
d\left(S, S^{\prime}\right)=\max \left\{\delta\left(S, S^{\prime}\right), \delta\left(S^{\prime}, S\right)\right\}
$$

where

$$
\begin{aligned}
& \delta\left(S, S^{\prime}\right)=\sup _{x \in S} \delta\left(. x, S^{\prime}\right) \\
& \delta\left(x, S^{\prime}\right)=\inf _{x, \in S}\left\|x-x^{\prime}\right\|
\end{aligned}
$$

and $\|\cdot\|$ denotes the Euclidean norm. In order to show that the map $\kappa$ associated with a closed convex set $K$ and an affine transformation $\tau$ is Lipschitzian with constant $B$ it suffices to show that

$$
\delta\left(\kappa(y), \kappa\left(y^{\prime}\right)\right) \leqq B\left\|y-y^{\prime}\right\|
$$

whenever $\kappa(y)$ and $\kappa\left(y^{\prime}\right)$ are nonempty.

Now assume that $K$ is a closed convex polyhedron $P$, let $L$ be the lineality space of some nonempty section $\kappa(y)$, i.e., the largest subspace of $E^{n}$ for which a translate is contained in $\kappa(y)$, and let $L^{*}$ be the subspace of $E^{n}$ orthogonal to $L$. It is easily shown that $L$ is in fact the lineality space of every nonempty section $\kappa(y)$ and is contained in the lineality space of $P$. Thus $P$ is the vector sum of $L$ and the polyhedron $P^{*}=P \cap L^{*}$ and each nonempty section $\kappa(y)$ is the vector sum of $L$ and a pointed polyhedron $\kappa^{*}(y)=\kappa(y) \cap L^{*}$. Since any two nonempty sections $\kappa(y)$ and $\kappa\left(y^{\prime}\right)$ are parallel cylinders, the distance between them can be realized as the distance between points of the sets $\kappa^{*}(y)$ and $\kappa^{*}\left(y^{\prime}\right)$. Also, if we denote the restriction of $\tau$ to $L^{*}$ by $\tau^{*}$, then $\kappa^{*}(y)=\tau^{-1}(y) \cap L^{*} \cap P=\tau^{*-1}(y) \cap P^{*}$. Thus for the rest of the proof we may restrict our attention to $P^{*}$, the affine transformation $\tau^{*}$, and the associated map $\kappa^{*}$, or equivalently we may as well assume that each nonempty section $\kappa(y)$ of $P$ is a pointed polyhedron.

Note also that every nonempty section $\kappa(y)$ contains the same cone part $C$, i.e., the largest closed convex cone with apex at the origin for which a translate is contained in $\kappa(y)$. Let $v(y)$ denote the set of vertices of $\kappa(y)$ and let con $v(y)$ denote the convex hull of $v(y)$. Since we have assumed that every nonempty $\kappa(y)$ is a pointed polyhedron, the cone $C$ is also pointed, $v(y)$ is nonempty, and $\kappa(y)=\operatorname{con} v(y)+C$.

We now assert that

$$
\delta\left(\kappa(y), \kappa\left(y^{\prime}\right)\right)=\delta\left(\operatorname{con} v(y), \kappa\left(y^{\prime}\right)\right)
$$

whenever $\kappa(y)$ and $\kappa\left(y^{\prime}\right)$ are nonempty. Consider any point $x$ of $\kappa(y)$. Since $\kappa(y)=\operatorname{con} v(y)+C$, there exist $x_{b} \in \operatorname{con} v(y)$ and $q \in C$ such that 
$x=x_{b}+q$. The distance $\delta\left(x_{b}, \kappa\left(y^{\prime}\right)\right)$ is achieved as the distance $\left\|x_{b}-x^{\prime}\right\|$ for some $x^{\prime}$ in $\kappa\left(y^{\prime}\right)$. Since we also have that $\kappa\left(y^{\prime}\right)=\kappa\left(y^{\prime}\right)+C$, the point $x^{\prime}+q$ is in $\kappa\left(y^{\prime}\right)$. Hence $\delta\left(x, \kappa\left(y^{\prime}\right)\right) \leqq\left\|x-\left(x^{\prime}+q\right)\right\|=\left\|x_{b}-x^{\prime}\right\| \leqq$ $\delta\left(\operatorname{conv}(y), \kappa\left(y^{\prime}\right)\right)$, from which the above assertion follows easily.

Considered as a function of $x, \delta\left(x, \kappa\left(y^{\prime}\right)\right)$ is a finite continuous convex function. It follows that it achieves a maximum over the bounded convex polyhedron con $v(y)$ at one of the points of $v(y)$. Thus

$$
\delta\left(\kappa(y), \kappa\left(y^{\prime}\right)\right)=\delta\left(\operatorname{con} v(y), \kappa\left(y^{\prime}\right)\right)=\delta\left(v(y), \kappa\left(y^{\prime}\right)\right),
$$

from which it follows immediately that

$$
\delta\left(\kappa(y), \kappa\left(y^{\prime}\right)\right) \leqq \delta\left(v(y), v\left(y^{\prime}\right)\right) .
$$

To complete the proof of the "only if" part of the theorem, we will show that $\delta\left(v(y), v\left(y^{\prime}\right)\right)$ satisfies a Lipschitz condition, i.e. there exists a constant $B$ independent of $y^{0}, y^{\prime}$, and the choice of point $x^{0} \in v\left(y^{0}\right)$ such that

$$
\delta\left(x^{0}, v\left(y^{\prime}\right)\right) \leqq B\left\|y^{0}-y^{\prime}\right\|
$$

whenever $\kappa\left(y^{0}\right)$ and $\kappa\left(y^{\prime}\right)$ are nonempty. Recall that $\tau$ is an affine transformation of $E^{n}$ onto $E^{n-i}$ taking the polyhedron $P$ onto another polyhedron, say $Q$, and $x^{0}$ is a vertex of the section $\kappa\left(y^{0}\right)$. The Lifting Theorem and related propositions of [4] assert that under these conditions there is a continuous piecewise linear map $\tau^{*}$ of $Q$ into $P$ such that

(i) $\tau^{*}(y)$ is a vertex of $\kappa(y)$ for every $y$ in $Q$,

(ii) $\tau^{*}(Q)$ is the union of closed faces of $P$ which are of the same dimension as $Q$ and on which $\tau$ acts one-to-one, and

(iii) $\tau^{*}\left(y^{0}\right)=x^{0}$.

The line segment joining $y^{0}$ and any other point $y^{\prime}$ of $Q$ is taken by $\tau^{*}$ into a polyhedral path on $P$ with nodes $x^{0}, x^{1}, \cdots, x^{r}$, say. Each segment $\left[x^{i}, x^{i+1}\right]$ of this path is contained in some face of $P$ on which $\tau$ acts one-to-one. For each face $F_{k}$ of $P$ on which $\tau$ acts one-to-one there is a constant $B_{k}$ such that

$$
\left\|x-x^{\prime}\right\| \leqq B_{k}\left\|\tau(x)-\tau\left(x^{\prime}\right)\right\| \quad \text { for all } x, x^{\prime} \text { in } F_{k} .
$$

Since there are only finitely many faces of $P$,

$$
\left\|x^{i+1}-x^{i}\right\| \leqq B\left\|\tau\left(x^{i+1}\right)-\tau\left(x^{i}\right)\right\|
$$

where $B$ is the largest of the $B_{k}$. Since

it follows that

$$
\left\|y^{\prime}-y^{0}\right\|=\sum_{i=0}^{r-1}\left\|\tau\left(x^{i+1}\right)-\tau\left(x^{i}\right)\right\|
$$




$$
\left\|x^{r}-x^{0}\right\| \leqq B\left\|y^{\prime}-y^{0}\right\|,
$$

which implies (1) since $x^{r} \in v\left(y^{\prime}\right)$.

As observed in the introduction, the validity of Theorem 1 in no way depends on the use of the Euclidean norm. We remark without proof that the distance $d\left(\kappa(y), \kappa\left(y^{\prime}\right)\right)$ is actually piecewise linear in the joint variables $y$ and $y^{\prime}$ if the $l_{1}$-norm (or any norm with polyhedral unit ball) is used in the domain of $\tau$. The Lipschitzian property of $d\left(\kappa(y), \kappa\left(y^{\prime}\right)\right)$ might seem to be a trivial consequence of this observation; but a proof of the theorem based on this idea would still require all the arguments used above in one disguise or another.

3. Property $R_{j}$ implies polyhedral. We start by reviewing some definitions and results concerning polyhedra established by Klee in [1].

Definition 2. A subset $K$ of $E^{n}$ is said to be polyhedral at a point $p$ of $K$ if there exists a neighborhood of $p$ relative to $K$ which is a convex polytope. A subset $K$ of $E^{n}$ is said to be boundedly polyhedral if its intersection with any polytope is again a polytope.

Note that any subset of $E^{n}$ contained in a flat $F$ is polyhedral at every point of its interior relative to $F$. Also, a closed convex subset of $E^{n}$ is boundedly polyhedral if and only if it is polyhedral at each of its points. The two following theorems are slight variations of Theorems (4.1) and (4.7) of [1] containing the information relevant for our present purposes.

TheOREM 2. Suppose $K$ is a convex subset of $E^{n}, p \in K$, and $2 \leqq j \leqq n$. Then $K$ is polyhedral at $p$ if and only if for every $j$-dimensional flat $J$ through $p$ and every projection $\tau$ of $E^{n}$ onto $J$ the set $\tau K$ is polyhedral at $p$.

Theorem 3. Suppose $K$ is a convex subset of $E^{n}, p \in K$, and $2 \leqq j \leqq n$. Then $K$ is boundedly polyhedral (respectively polyhedral) if and only if every section of $K$ by a $j$-dimensional flat is boundedly polyhedral (respectively polyhedral).

Just as the property "polyhedral at a point" localizes the property "polyhedral," the property $\mathfrak{l}_{j}$ may be localized as follows:

Definition 3. A subset $K$ of $E^{n}$ has property $\mathfrak{R}_{j}$ at a point $p$ of $K$ if there exists a constant $B$ such that

$$
d[(N+p) \cap K,(N+q) \cap K] \leqq B\|p-q\|
$$

for all $q$ in $K$ and all $j$-dimensional subspaces $N$ of $E^{n}$. The subset $K$ has property $\mathfrak{R}_{N}$ at $p$ if (2) holds for all $q$ in $K$ where $B$ depends on $p$ 
and the particular subspace $N$. The subset $K$ has property $\mathfrak{R}_{N}$ if (2) holds for all $p$ and $q$ in $K$ where $B$ depends on $N$ only.

Proposition 1. Suppose $K$ is a closed convex subset of $E^{n}$ and $p \in K$. Then $K$ is polyhedral at $p$ if and only if $K$ is $\Omega_{n-1}$ at $p$.

Proof. Suppose $K$ is not polyhedral at $p$, then by Theorem 2 there is a projection $\tau(K)$ on a 2 -flat $G$ through $p$ which is not polyhedral at $p$. For 2-dimensional convex sets the proposition is obvious; there exists some one-dimensional subspace $l, l+p \subset G$, for which $\tau(K)$ is not $\Omega_{l}$ at $p$. It readily follows that $K$ is not $\Omega_{H}$ at $p$, where $H$ is the hyperplane $\tau^{-1}(l)$.

Proposition 2. Suppose $K$ is a closed convex subset of $E^{n}$ and $1 \leqq j \leqq n-1$. Then $K$ is boundedly polyhedral if $K$ is $\Omega_{j}$ at $p$ for all $p$ in $K$.

Proof. Suppose that $K$ has property $\Omega_{j}$ at $p$ for each $p$ in $K$. If the dimension $k$ of $K$ is almost 1 , then $K$ is in fact polyhedral. Thus suppose $k$ is at least 2 and write

$$
j^{\prime}=\max \{1, j-n+k\} \quad k^{\prime}=j^{\prime}+1
$$

so that $2 \leqq k^{\prime} \leqq k$. Consider any point $p$ of $K$, any section $K^{\prime}$ of $K$ of dimension $k^{\prime}$ through $p$, and any subspace $J^{\prime}$ such that $J^{\prime}+p$ is a flat of dimension $j^{\prime}$ contained in the affine hull of $K^{\prime}$. The numbers $j^{\prime}$ and $k^{\prime}$ are so defined that there is a subspace $J$ of dimension $j$ such that

$$
\left(J^{\prime}+q\right) \cap K^{\prime}=(J+q) \cap K \quad \text { for all } q \text { in } K^{\prime} .
$$

Since $K$ has property $\Omega_{J}$ at $p$ it follows that $K^{\prime}$ has property $\Omega_{J^{\prime}}$, at $p$, i.e., if $K^{\prime}$ is considered only as a subset of its affine hull it has property $\Omega_{j^{\prime}}$, at $p$. Since $k^{\prime}=j^{\prime}+1$, Proposition 1 applies, and hence $K^{\prime}$ is polyhedral at $p$. Since $p$ and $K^{\prime}$ are arbitrary it follows from Theorem 3 that $K$ is boundedly polyhedral.

NOTE ADDED IN PROOF. We remark that Proposition 1 remains valid if $\Omega_{n-1}$ is replaced by $\Omega_{j}$ for arbitrary $j, 1 \leqq j \leqq n-1$. The proof makes use of slightly different arguments.

Also, in connection with projections of simplices in vector groups, William R. Emerson has independently obtained a weaker version of Theorem 1, viz.: Suppose $K$ is a closed bounded convex subset of $E^{n}$. Then $K$ is a polyhedron if and only if it is $\ell_{1}$.

Proof OF The "IF" PART OF Theorem 1. Suppose $K$ is a closed convex subset of $E^{n}$ with property $\mathfrak{R}_{j}$ for some $j$ between 1 and $n-1$. By Proposition 2, $K$ is boundedly polyhedral. Thus, $K$ is the intersection of a minimal set of halfspaces $\left\{H_{i}^{+}\right\}$in the affine hull $M$ of $K$, 
so that for each $H_{i}^{+}$there is a point $p_{i}$ in the interior of $H_{i} \cap K$ relative to $H_{i}$, where $H_{i}$ is the hyperplane in $M$ bounding $H_{i}^{+}$. Let $h_{i}$ be the unit vector in $M$ perpendicular to $H_{i}$. If $K$ is not polyhedral, there is a subsequence $\left\{h_{i(k)}\right\}$ of $\left\{h_{i}\right\}$ converging to, but never achieving, a vector $h_{0}$. Let $J_{0}$ be a subspace of $E^{n}$ of dimension $j$ orthogonal to $h_{0}$. Then the angles between $J_{0}+p_{i(k)}$ and $H_{i(k)}$ converge to, but never achieve, zero. It follows that no one Lipschitz constant $B$ will work uniformly in the condition $\Omega_{J_{0}}$ at all points $p_{i(k)}$ of $K$. This contradiction completes the proof.

4. Some extensions. In this section we examine some of the consequences of property $\mathfrak{R}_{j}$ when $K$ is not closed or not convex. In particular, removing the closed conditions yields the following lukewarm generalization of Theorem 1. (A subset $K$ of $E^{n}$ is a wholefaced polyhedron [3] if it is the intersection of finitely many closed and open halfspaces.)

Theorem 4. Suppose $K$ is a convex subset of $E^{n}$ and $1 \leqq j \leqq n-1$. Then $K$ is a wholefaced polyhedron if and only if $K$ has property ${ }_{i j}$.

The above theorem follows immediately from Theorem 1 and the proposition below. The proof of this proposition is omitted since it is an easy consequence of the continuity of the Hausdorff distance; however, it is not completely trivial since a nonempty section of $K$ may correspond to an empty section of $S$.

Proposition 3. Suppose $K$ is a closed subset of $E^{n}$ and $S$ is a dense subset of $K$. Then $S$ has property $\mathfrak{R}_{N}$ with associated constant $B$ if and only if both the following hold:

(i) The closure of the section $(N+p) \cap S$ is the section $(N+p) \cap K$ for every $p$ in $S$.

(ii) $K$ has property $\mathfrak{R}_{N}$ with the same associated constant $B$.

We take Proposition 3 as justification for restricting the remaining discussion to closed sets with propery $\ell_{j}$. For any $j, 1 \leqq j \leqq n-1$, the following closed sets in $E^{n}$ have property $\ell_{j}$ :

(i) any closed polyhedron,

(ii) any finite set,

(iii) any set obtained as the vector sum of a subspace of $E^{n}$ and an arbitrary closed subset of a line.

Item (i), of course, is the burden of $\$ 2$. Items (ii) and (iii) are easily verified directly. It can be shown that any closed set with property $\ell_{j}, 1 \leqq j \leqq n-1$, must be the intersection of sets of type (iii), and if it is not a set of type (i) or (ii) it must be quite peculiar. We con- 
jecture that (i), (ii), and (iii) are the only kinds of closed sets with property $\mathfrak{R}_{j}, 1 \leqq j \leqq n-1$.

\section{REFERENCES}

1. V. Klee, Some characterizations of convex polyhedra, Acta Math. 102 (1959), 79-107.

2. - Polyhedral sections of convex bodies. Acta Math. 103 (1960), 243-267.

3. T. S. Motzkin, Beiträge zur Theorie der Linearen Ungleichungen, Doctoral Dissertation, University of Basel, Azriel, Jersualem, 1936.

4. D. W. Walkup and R. J.-B. Wets, Lifting projections of convex polyhedra, Pacific J. Math. 28 (1969), 465-475.

Boeing Scientific Research Laboratories, Seattle, Washington 